\title{
Educación Inclusiva y Enseñanza Superior desde la mirada de estudiantes con Diversidad Funcional
}

\author{
Inclusive Education and Higher Education from the Viewpoint of Students with Functional Diversity \\ Educação inclusiva e ensino superior a partir da perspectiva dos estudantes com diversidade funcional
}

Anabel Moriña* (http://orcid.org/0000-0002-0852-7523)

Facultad de Ciencias de la Educación, Universidad de Sevilla, España Almudena Cotán Fernández** (http://orcid.org/0000-0003-0362-4348)

Universidad Isabel I, España
Recibido: $23-02-17$

Revisado: 22-04-17

Aceptado: $07-06-17$

Publicado: 18-06-17
RESUMEN. Este artículo presenta los resultados de una investigación en la que se analizaron los facilitadores y obstáculos que tres estudiantes con diversidad funcional identificaron durante su trayectoria en la universidad. Concretamente, se estudió cómo el profesorado había podido contribuir o no a la inclusión social y educativa. A partir del método biográfico-narrativo se pretendía dar voz a personas con diversidad funcional. Para la recogida de información se hizo uso de diversos instrumentos cualitativos: entrevistas biográficas, entrevistas focalizadas, entrevistas semiestructuradas, fotografías, autoinformes, línea de vida, diario de campo de la investigadora, notas de campo de la investigadora, observaciones, correos electrónicos y la técnica "Un día en la vida de...". Se llevó a cabo un análisis narrativo que permitió construir tres historias de vida. En concreto, los resultados de este trabajo, a través de las narraciones de Marper, Andrés y Rafa, se centran en analizar cómo el profesorado contribuía o no a la inclusión de los estudiantes y cuáles eran las principales barreras que identificaban referidas a éste. Entre las principales conclusiones cabe resaltar cómo algunos docentes no estaban suficientemente formados para responder a las necesidades de los estudiantes o cómo, en ocasiones, no se realizaban los ajustes necesarios para el aprendizaje y participación de los estudiantes.
Palabras claves:

Enseñanza

Superior

Diversidad

Funcional, Inclusión,

Profesorado, Historia de Vida. 
ABSTRACT. This article presents the results of a research that sought to analyze the facilitators and obstacles that three students with functional diversity identified during their career in the university, namely how teachers had been able to contribute to their social and educational inclusion. From the biographical-narrative method was intended to give voice to people with functional diversity. For the collection of information, a variety of qualitative instruments were used: biographical interviews, focused interviews, semistructured interviews, photographs, self-reports, lifeline, researcher's field diary, Emails and the technique "A day in the life of ..." A narrative analysis was carried out that allowed to construct three histories of life. In particular, the results of this work, through the narratives of Marper, Andrés and Rafa, focus on analyzing how teachers contributed or not to the inclusion of students and what were the main barriers that identify referred to it. Among the main conclusions, it should be pointed out that some teachers were not sufficiently trained to respond to the needs of the students or because, sometimes, the necessary adjustments were not made for student learning and participation.
Key words:

Higher

Education,

Functional

Diversity,

Inclusion,

Teachers,

Life History.

RESUMO. Este artigo apresenta os resultados de uma pesquisa onde foram analisados os facilitadores e os obstáculos que três estudantes com diversidade funcional identificaram durante a sua carreira na universidade, especificamente na forma como os professores tinham sido capazes de contribuir ou não a sua inclusão social e educacional. A partir do método biográfico-narrativo pretendeu-se dar voz às pessoas com diversidade funcional. Para a coleta de informações utilizou-se vários instrumentos qualitativos: entrevistas biográficas, entrevistas focalizadas, entrevistas semi-estruturadas, fotografias, autorelatórios, linha de tempo, diário de campo da pesquisadora, notas de campo da

Palavraschave: ensino superior, diversidade funcional, inclusão, professorado, história de vida. pesquisadora, observações, e-mails e a técnica "um dia na vida de ...". Foi realizada uma análise narrativa que permitiu construir três histórias de vida. Especificamente, os resultados deste trabalho, através das narracões de Marper, Andrew e Rafa, concentramse em analisar como os professores contribuíram ou não na inclusão dos estudantes e quais foram as principais barreiras identificadas em relação a este. Entre as principais conclusões destaca como alguns professores não foram suficientemente treinados para atender as necessidades dos estudantes ou como, às vezes, não foram feitos os ajustes necessários para a aprendizagem e participação dos alunos.

El acceso a la universidad de personas con diversidad funcional es un derecho reconocido legalmente (Yssel, Pak \& Beilke, 2016). Por ejemplo, en España se ha avanzado en este sentido, con el Real Decreto Legislativo 1/2013 (Boletín Oficial del Estado [BOE], 2013) de derechos de las personas con diversidad funcional y su inclusión social. En éste se establece que la educación inclusiva ha de formar parte del proceso de atención integral de las personas con diversidad funcional, impartiéndose con los recursos, apoyos y ajustes que desde la legislación se considere necesarios. Asimismo, también la Ley Orgánica 4/2007 (BOE, 2007) de Universidades expresa el imperativo de responder a las necesidades 
y demandas de este colectivo así como adoptar medidas que garanticen, entre otros aspectos, la adaptación de las pruebas de acceso, su permanencia en los estudios universitarios, exención de tasas, aumento de becas y ayudas al estudio, creación de espacios arquitectónicos accesibles, adaptación de los aspectos formativos y la integración plena de los estudiantes (Aguirre \& Miguel, 2011; Rodríguez \& Álvarez, 2014; Vander Kloet, 2015).

Aunque existe esta normativa, es reconocido el vacío legal en su implementación, ya que, en ocasiones, las prácticas en la universidad no promueven procesos inclusivos. Diversos trabajos que dan voz al alumnado, se han hecho eco de esta realidad, y denuncian que los estudiantes tienen que recorrer una carrera de obstáculos que, en muchas ocasiones, genera un abandono prematuro de los estudios universitarios (Fuller, Bradley \& Healey, 2004; Moswela \& Mukhopadhyay, 2011; Mullins \& Pride, 2013; Novo, Muñoz \& Calvo, 2011; Shaw, 2009; Strauss \& Sales, 2010). Estos trabajos comparten la idea de que, aunque las instituciones de enseñanza superior suponen una oportunidad y pueden empoderar a estos estudiantes, aún queda un largo camino por recorrer para que esta institución sea inclusiva (Alcain, \& Medina, 2017; Dalmau, Llinares \& Sala, 2013; Denhart, 2008; Fuller et al., 2004; Gibson, 2012; Hopkins, 2011; Vickerman \& Blundell, 2010). De hecho, como Thomas (2016) ha concluido, no es suficiente con garantizar el acceso, son también necesarios políticas y planes para lograr la permanencia y éxito en los estudios universitarios.

No obstante, es preciso reconocer que paulatinamente hay un mayor compromiso por parte de las universidades para caminar hacia la inclusión. En este contexto, las iniciativas han de tener un triple compromiso: la generación de un conocimiento crítico, el desarrollo profesional y la intervención social (Rodríguez \& Álvarez, 2014; Salvador, 2003), exigiendo el cumplimiento de estándares de calidad, en el que la atención al alumnado con diversidad funcional y la respuesta a sus necesidades y demandas sea uno de ellos (Alonso \& Díez, 2008).

En este sentido, actualmente en España, las políticas educativas y de inclusión que se están llevando a cabo inciden de forma satisfactoria y positiva en la formación de los estudiantes con diversidad funcional en las universidades (Cerrillo, Izuzquiza \& Egido, 2013). Sin embargo, siguen existiendo problemas de acceso a la educación y de permanencia en las aulas durante su trayectoria académica que limita el proceso de aprendizaje (Foreman, Dempsey, Robinson \& Manning, 2001; Vickerman \& Blundell, 2010).

Trabajos como los de Castro de Paz, Llorca, Álvarez y Álvarez (2006) o González, Guzmán, Sánchez y González (2006) resaltan las barreras curriculares como obstáculo principal. Para la inclusión del alumnado se podrían precisar ajustes razonables, como uso de metodologías diversas, recursos que faciliten el acceso a los contenidos, tiempo extra o adaptación de las pruebas de evaluación. Para todo ello, la formación del profesorado se contempla como imprescindible. De hecho, es conveniente esta formación, ya que como Doktor (2010) y Ruiz Hidalgo (2015) indican, el docente puede ayudar a mejorar la experiencia de aprendizaje del alumnado. Sin embargo, hay trabajos como los de Borland y James (1999), Fuller, Healey, Bradley y Hall (2005), Hopkins (2011), Moriña (2017a) o Moriña, Cortés y Molina (2015), que concluyen que el profesorado es la principal barrera Identificada por los estudiantes, haciendo especial hincapié en la necesidad de formación y sensibilización del profesorado como vehículo conductor para ofrecer una actitud empática, abierta y flexible ante las necesidades del alumnado. 
En este sentido, los currículos rígidos y no inclusivos (Hopkins, 2011) dificultan el acceso a los contenidos y participación en las aulas universitarias. Autores como Fuller et al., (2005) o Wessel, Jones, Blanch y Markle (2015), plantean que esta situación hace que los estudiantes tengan dificultades para conseguir la realización de modificaciones curriculares: material por adelantado, grabación de las clases, adaptaciones de exámenes, metodologías diversas, etc. En los planes de estudio, estos inconvenientes, en numerosas ocasiones, se asocian con la idea de que la realización de modificaciones curriculares posicionaría en una situación de ventaja al estudiante con diversidad funcional frente a sus compañeros, o que éstas supondrían bajar el nivel curricular de la materia y, por lo tanto, la no adquisición de los objetivos de la misma (Riddell, Tinklin \& Wilson, 2005; Tinklin, Riddell \& Wilson, 2004).

Estos trabajos, en definitiva, muestran la brecha existente entre las políticas y la práctica real al presentar evidencias significativas que impiden, en ocasiones, la participación en el aprendizaje de las personas con diversidad funcional. Por ello, numerosos estudios plantean la necesidad de formación del profesorado para mejorar las estrategias de enseñanza-aprendizaje frente al alumnado (Debrand \& Salzberg, 2005; Doughty \& Allan, 2008; Healey, Fuller, Bradley \& Hall, 2006; Leyser \& Greenberger, 2008; Murray, Lombardi \& Wren, 2011; Teachability, 2002). En el caso nacional, destacar, por ejemplo, las propuestas formativas realizadas por Escandell et al. (2008), Guasch y Hernández (2011) o Moriña (en prensa), quienes plantean la creación de material de formación específico para formar a los docentes desde planteamientos metodológicos diversos y accesibles. Sin embargo, sigue siendo necesario plantear más procesos formativos, así como ofrecer más recursos para garantizar el desarrollo de los mismos.

Por todo ello, consideramos que la educación inclusiva ha de responder a la diversidad, debiendo de ser este un tema que necesita contemplarse en las agendas de todas las universidades, siendo un principio de debate, sensibilización y formación (Novo et al., 2011). De hecho, es necesario e imprescindible que el profesorado tenga la oportunidad y el espacio para formarse y dar respuestas educativas a la diversidad.

En definitiva, el propósito principal de este artículo es presentar las narraciones de tres estudiantes referidas a sus experiencias en la universidad y cómo el profesorado ha podido contribuir o no a su inclusión social y educativa.

\section{MÉTODO}

Diseño

Los resultados presentados en este artículo están vinculados a la tesis doctoral (Cotán, 2015), que se desarrolló en el marco de un proyecto de investigación financiado por el Ministerio de Economía y Competitividad de España (ref. EDU2010-16264) ${ }^{1}$. El propósito era identificar, desde la perspectiva de los estudiantes con diversidad funcional, las barreras y ayudas encontradas en sus trayectorias universitarias. Concretamente, en esta tesis doctoral analizamos los obstáculos, facilitadores y propuestas de mejora que tres estudiantes identificaban en sus trayectorias formativas en la universidad, referidas, especialmente, al profesorado.

1 Proyecto I+D+l "Barreras y ayudas que los estudiante con discapacidad identifican en la Universidad" (Ref. Edu2010-16264) financiado por el Ministerio de Ciencia e Innovación y bajo la dirección de la Dra. Anabel Moriña. 
La metodología de investigación ha sido la biográfico-narrativa (Moriña, 2017b). El motivo principal por el cual hemos seleccionado este tipo de método es porque permite hacer oír la voz de los alumnos en el contexto universitario. En esta investigación no solo nos interesa la construcción de nuevos conocimientos, sino también poder desvelar y ofrecer el pensamiento y las experiencias de los estudiantes con diversidad funcional, así como las circunstancias que condicionan y dan forma al ambiente educativo universitario.

\section{Contexto}

Esta investigación se desarrolló en una universidad pública del sur de España. A través de las narraciones que surgen de las historias de vida, intentaremos conocer y analizar los diferentes contextos universitarios en los que los estudiantes están inmersos. En cualquier caso, el estudio de estos contextos universitarios ofrece una explicación de la cultura que envuelven a los estudiantes realizando una deconstrucción de la realidad a raíz de sus experiencias, reinterpretando los hechos sucedidos y transcurridos a lo largo de su experiencia.

Concretamente, el contexto universitario en el que nos centramos y que rodeó las narraciones fue la facultad de Derecho, Informática y Periodismo. A partir de esto y de sus narraciones contextualizadas intentamos buscar información sobre cómo el centro, sus características, ubicación, líneas de comunicación y transporte, etc. y todo lo relacionado con la institución y aulas universitarias influyeron en las experiencias de los estudiantes, identificándolos como obstáculos o facilitadores.

\section{Participantes}

El acceso a los participantes de este estudio se hizo a través de la Unidad de Atención a Estudiantes con discapacidad de la Universidad en la que tiene lugar el estudio. Para la selección de los participantes nos hemos basado en el procedimiento establecido por Goetz y LeCompte (1988), denominado "selección basada en criterios", donde la selección se encuentra determinada previamente por unos criterios establecidos. En este sentido, y siguiendo a Patton (1987) y Pujadas (2002), hemos realizado un muestreo selectivo atendiendo a unos rasgos, criterios y características relevantes: estudiante con diversidad funcional accesibilidad, participación, disponibilidad para participar, relevancia, voluntariedad e interés o necesidad.

En esta investigación participaron un total de tres estudiantes incluidos en la muestra de los 44 estudiantes que participaron en la investigación mencionada. Los tres participantes estaban estudiando diferentes titulaciones: Derecho, Ingeniería y Periodismo. Respecto a su permanencia en la universidad, en el momento de la obtención de los datos, el primero de los estudiantes llevaba diez años en la universidad, el segundo seis y el tercero cuatro.

La primera informante que, por anonimato y protección de datos vamos a proceder a darle un nombre ficticio², es Marper, con parálisis cerebral, en el momento de la recogida de datos, contaba con 38 años, su permanencia en la universidad era de 11 años (desde el curso 2001/2002 hasta el curso 2012/2013) y se encontraba cursando las últimas asignaturas para finalizar el Grado de Derecho.

2 Siguiendo los principios éticos por los que nos hemos regido en todo momento en la investigación, los nombres empleados en este trabajo han sido seleccionados por los participantes. 
En cuanto al segundo de los participantes, Andrés, en el momento de la recogida de la información tenía 27 años de edad e inició sus estudios en Ingeniería ${ }^{3}$, correspondiente al plan del 97 en el año 2006, cambiándose, años más tarde, al actual Grado de la misma titulación. Su permanencia en la universidad era de seis años, encontrándose en segundo grado. Su diversidad funcional era visual.

Y, para finalizar, el último de los participantes con quien hemos mantenido contacto para realizar la historia de vida en profundidad, es Rafa. Con 20 años de edad y afectado de distrofia muscular de Duchenne, estaba en segundo curso del Grado de Periodismo cuyo curso académico de acceso se sitúa en el año 2011/2012 y, llevando una duración total de cuatro años en la universidad.

\section{Tabla 1}

Perfil de los participantes

$\begin{array}{cccccc}\text { Nombre } & \text { Edad } & \text { Titulación } & \text { Curso } & \begin{array}{c}\text { Años en la } \\ \text { universidad }\end{array} & \text { Diversidad } \\ \text { Marper } & 38 & \text { G. Derecho } & 4^{\circ} & 10 & \begin{array}{c}\text { Física } \\ \text { (Parálisis Cerebral) } \\ \text { Visual } \\ \text { (Ceguera) } \\ \text { Rafa }\end{array} \\ 27 & \text { G. Ingeniería } & 2^{\circ} & 6 & \begin{array}{c}\text { Física } \\ \text { (distrofia muscular de Duchenne) }\end{array}\end{array}$

\section{Instrumentos de Recolección de Datos}

La recogida de datos en este trabajo de investigación se ha basado en diversas técnicas cualitativas: entrevistas biográficas, entrevistas focalizadas, entrevistas semiestructuradas, fotografías, autoinformes, línea de vida, diario de campo de la investigadora, notas de campo de la investigadora, observaciones, correos electrónicos y la técnica "Un día en la vida de...".

\section{Procedimiento}

Para acceder a dicha muestra, y teniendo en cuenta la Ley de Protección de Datos II del REAL DECRETO 1720/2007, de 21 de diciembre, por el que se aprueba el Reglamento de desarrollo de la Ley Orgánica 15/1999, de 13 de diciembre, de protección de datos de carácter personal, el primer paso fue contactar (a través de una entrevista telefónical con la responsable de la Unidad de Atención a Estudiantes con Discapacidad y hacerle conocer nuestro proyecto de investigación y el objeto del contacto.

Una vez realizado este paso, fue esta quien intermedió para solicitar la participación de los estudiantes, enviándole un email en el que se adjuntaba la carta de presentación elaborada por el grupo de investigación BUDA ${ }^{4}$, donde se explicaba toda la investigación y se les invitaba a participar.

3 Según los criterios éticos de nuestra investigación y por petición expresa de Andrés, en esta investigación no aparecerá ningún nombre real ni dato que pueda identificarlo. 4 BUDA es el acrónimo del equipo de investigación coordinado por la Dra. Anabel Moriña cuyo significado es Barreras, Universidad, Discapacidad y Ayuda (por una universidad inclusiva). 
El análisis de los datos se ha realizado de forma paralela a la recogida de los datos, ya que desde el acceso al campo de trabajo comenzamos a transcribir y analizar la información recogida. A partir de este trabajo progresivo, planteamos nuevos temas o contenidos para seguir profundizando en las historias de vida. Siguiendo a Bertaux (2005), consideramos que el análisis de los datos no es el algo que surja únicamente en el momento de su realización (Moriña, 2017b), sino que desde el inicio de nuestro trabajo nos hemos cuestionado, orientado y reflexionado, por lo que podemos afirmar que durante la recogida de datos, el análisis nos ha aportado información sobre la propia trayectoria del proyecto: dificultades, aspectos de mejora, temas por tratar, etc.

En esta investigación hemos realizado dos tipos de análisis: narrativo y estructural (Bolívar, Domingo \& Fernández, 2001). Sin embargo, para este artículo se ha utilizado exclusivamente el análisis narrativo. La elaboración e interpretación del relato comenzó por explorar y conocer las historias en profundidad buscando varias perspectivas y comprensiones para organizar la historia en temas centrales (Denzin \& Lincoln, 1998). La presentación de los datos de la historia se focalizó en momentos esenciales e importantes para los protagonistas interpretando los significados de los hechos, relacionándolos con el contexto social, cultural y político en el que se desarrolló.

\section{Consideraciones Éticas}

Desde el punto de vista de nuestro trabajo de investigación y, en concreto, desde la investigación biográfico-narrativa, planteamos determinadas consideraciones éticas (Hammersley \& Atkinson, 2007; Opazo, 2011; Pacheco, 2012; Richaud, 2007; Sikes, 2006; Stutchbury \& Fox, 2009; Vain, 2011) que debemos tener en cuenta en la recogida de la información y, posteriormente, en su publicación. Por ello, durante todo el proceso de nuestro trabajo todos los participantes tuvieron acceso a un consentimiento informado para formar parte del estudio. Dicho consentimiento fue firmado en la primera toma de contacto en la que se le informó del procedimiento y tratamiento que tendrían la información recopilada.

Durante todo el proceso del estudio, el estudiante ha tenido acceso a la información recopilada pudiendo modificarla o suprimirla si así lo estimase oportuno. Además, se les informó que en el caso de no querer continuar adelante con el estudio una vez iniciado, sus datos no serían tenidos en cuenta para el análisis pasando a ser destruidos y no publicados.

\section{RESULTADOS}

En este apartado se presentan los relatos obtenidos de los tres participantes en la investigación: Marper, Andrés y Rafa. Para ello y siguiendo el orden cronológico en el que tomamos el contacto, en primer lugar presentamos la historia de vida de Marper, caracterizada por su espíritu luchador y de superación. En segundo lugar, la historia de vida de Andrés, donde su eje principal se centra en las barreras surgidas en el contexto universitario y, por último, en tercer lugar, la historia de vida de Rafa, quien pasó de ser un estudiante tímido y reservado a convertirse en todo un periodista con un prometedor futuro. 


\section{La Historia de Vida de Marper}

Me llamo Marper, aunque he nacido en Sevilla me fui a vivir durante unos años al campo de Gibraltar, Algeciras. Pero siempre se dice que uno siempre vuelve a su tierra, por lo que actualmente resido en Sevilla, mi casa. Tengo 38 años y soy estudiante de último curso del Grado de Derecho. No ha sido un camino fácil, mis problemas vienen arraigados desde BUP y COU. En estos cursos, los profesores no me prestaban las ayudas necesarias y, al no tener finalizado Bachiller, no pude hacer Selectividad hasta los 25 años en la prueba de acceso a la Universidad (PAU) que, conseguí aprobarla y cumplí mi sueño: acceder a la Universidad.

Comencé mis estudios en el curso 2001/2002, en la facultad de Derecho. Mis comienzos no fueron fáciles, especialmente si traes a tus espaldas problemas como los que yo tuve en cursos inferiores (BUP y COU). No sabía cómo estudiar, organizarme, no encontré compañeros en la Facultad, tuve problemas con algunos profesores... pero también tuve muchas gratificaciones, ¿la más importante? La oportunidad de poder estudiar una carrera como Derecho.

Sin embargo, las principales barreras con las que me he encontrado han sido las metodologías y los proyectos docentes. La mayoría de los profesores se limitan a dictar en clase y a seguir un libro. La verdad, yo con el libro no me entero, es tanta materia... No sé cómo voy a estudiarlo... En ocasiones, ni el propio profesorado entiende los libros.

Hay profesores que llegan un día a clase y te indican que hay que hacer una actividad y, una vez finalizada la hora de clase, la recogen y se la llevan. A mí no me da tiempo, y tengo que finalizarlas en mi casa y, la verdad, me siento un poco mal, porque tengo que destinar tiempo en mi casa a realizarla y, mis compañeros, por ejemplo, no. Ese tiempo lo tienen para poder dedicarlo al estudio.

Bajo mi punto de vista, las clases, la verdad, es que son un poco aburridas porque yo llego y el profesor está dictando, mis compañeros copiando y, como yo no puedo seguir el ritmo del dictado, pues estoy atenta a lo que dice el profesor, pero, siendo sincera, es un poco aburrido.... Siempre es igual, no existe ningún tipo de dinámica por parte del profesorado, son clases muy monótonas, pero al final, no te queda más remedio y te acabas acostumbrando.

De todas formas, con los profesores casi no tengo relación, única y estrictamente la que se puede derivar de las clases, salvo con uno que me ha ayudado a hacer mi practicum. Es que jhay profesores y profesores! Por ejemplo, he tenido una profesora que se negó a realizarme el examen tipo test porque decía que era una discriminación respecto a mis compañeros ¡Discriminación! Si yo no quería menos materia, ni un examen más fácil ¡sólo quería el examen tipo test! Al final, tras estar un año pidiéndole la adaptación y con mucho esfuerzo, conseguí que me lo hiciera... Pero mi trabajo me costó.

También me he encontrado con otro profesor, ¿cómo podría definirlo? Es una persona que carece de empatía, o al menos así lo veo yo. He tenido que ir dos veces a su despacho a hablar con él, porque no comprendía bien sus clases ni su materia y me dijo en mi cara que no me entendía lo que decía cuando le hablaba, tampoco se preocupó por entenderme... Así que me quedé con las mismas dudas con las que entré en su despacho y con una indignación gigante. 
Yo creo que los profesores deberían evaluarse o formarse en pedagogía. Desde mi punto de vista considero que eso le ayudaría a responder a las necesidades de las personas con diversidad funcional, aunque quizás esté equivocada...

Pero bueno, solo ha sido con pocos profesores, la verdad es que la gran mayoría me ayuda. Quizás el fallo ha sido mío porque no he hablado desde el inicio de curso con los profesores, no les he contado mis necesidades, pero bueno, he ido durante el curso y la respuesta generalizada ha sido muy positiva: "No te preocupes, te hago el examen tipo test y te doy el tiempo que necesites".

La verdad es que estoy muy contenta con los profesores y ellos, yo creo, que conmigo también; lo único es que hay profesores que son más problemáticos que otros, pero eso es como todo. No obstante, creo que los profesores deben de estar formados para poder atender a la diversidad funcional, no sé si en Pedagogía o en otro ámbito, pero deben de estar formados. Las clases deben mejorarse, no han de ser tan magistrales y favorecer la comunicación dentro de ella. Únicamente existe una dirección de comunicación, la que el profesor indica a los alumnos.

Las actividades deberían estar adaptadas a las necesidades. Yo quiero poder terminar las actividades a la misma vez que mis compañeros o encontrar mecanismos alternativos para poder realizar las exposiciones. Y, por supuesto, fomentar el compañerismo entre los alumnos del centro.

\section{La Historia de Vida de Andrés}

Empezaré contando algo sobre mí. Me llamo Andrés, soy un chico de 27 años, nacido en un pueblo de la zona norte de Sevilla, aunque actualmente resido en la zona céntrica de esta bonita ciudad, en un piso compartido con mi hermano. Provengo de una gran familia, somos ocho hermanos, yo soy el sétimo. Mi familia lo es todo para mí.

Comencé la Universidad en el año 2006, ese nivel de estudio por lo que tanto había trabajado y me había esforzado. Sin embargo, no todo fueron luces en mi camino, también han existido sombras muy complicadas de pasar.

Mi inicio fue caótico, empezando desde la prueba de selectividad que tuve problemas con los recursos que me ofrecieron, lo que me provocó una desconcentración y nerviosismo enorme. La aprobé en setiembre y entré en la universidad al final de la fase de selección, por el mes de noviembre.

Sin embargo, me encontré con asignaturas que tenía que tener un $80 \%$ de presencialidad en las clases y a la fecha en la que entré eso ya era una misión imposible. Por lo que ese cuatrimestre lo di por perdido. El segundo cuatrimestre lo cogí con más fuerza, pero también resultó ser desastroso: solo aprobé una asignatura, lo que me garantizó la permanencia en los estudios.

En este cuatrimestre empezaron mis primeros problemas con la asignatura que me ha ido marcando a lo largo de mi trayectoria. El profesor se limitaba a impartir las clases con transparencias manuscritas a través de la lámpara de proyección, un soporte inaccesible para mí y, pese a solicitarle los apuntes para poder estudiarlos, lo único que encontré fue una negativa por su parte. 
Incluso un profesor llegó a decirme que no entendía por qué estaba allí o que no servía para estudiar esa titulación. Sin embargo, hice todo lo que pude por aprobar: fui a tutorías con dos compañeros míos, pero de nada sirvió, bueno sí, dividió nuestro grupo y aprobó a los que a él le pareció.

Desde mi punto de vista, puedo decir que el principal obstáculo con el que me he encontrado ha sido con los profesores. No han realizado bien las adaptaciones y, por lo tanto, no se ha dado respuesta a mis demandas ni a mis necesidades. Especialmente, tengo que destacar mi experiencia con el departamento problemático desde el primer año donde no solo se me privó del acceso a la información sino que también me cuestionó mi permanencia en los estudios.

Siempre ha habido una batalla con el tiempo extra en los exámenes. Una batalla que es absurda porque desde un punto de vista legal está estipulado que si hay alguna persona con diversidad funcional y esta tiene un problema se le debe de atender conforme a su problema, no conforme a un estándar o patrón preestablecido... Entonces, tenemos siempre la batalla de un tercio, dos tercios, tres tercios... El problema es que con un tercio no me daba a lo mejor tiempo de terminar por la complejidad visual de los materiales que había que consultar.

Después, a otros profesores les he tenido que pedir la adaptación del material y me han llegado a decir que estaba en una situación de ventaja respecto a mis compañeros e incluso profesores que no reconocían la diversidad funcional como tal y me decían que no iban a hacer más trabajo del que me corresponde.

En ciertas ocasiones me he sentido discriminado por la actitud de los profesores, no me han facilitado las herramientas ni recursos adecuados, como por ejemplo, los gráficos en relieve. Por lo que mi situación no era de ventaja frente a mis compañeros, sino todo lo contrario, estaba en desventaja porque no me estaban dando la oportunidad de poder acceder a la información y al material del examen: si no puedo ver el material del examen, no puedo hacerlo.

0, por ejemplo, las clases expositivas con ayuda del Power Point. Es una herramienta gráfica y visual que te resulta muy difícil acceder a ella y, si encima, el profesor se niega a facilitártelo peor aún. Te está discriminando porque no te está respetando tu derecho a la información, al que tenemos todos.

La verdad es que toda esta situación, en determinados momentos, ha llegado a desmotivarme y derrumbarme, porque cuando casi a diario estás escuchando "vete a otro sitio a estudiar", "vete de Erasmus y huye del problema" o "cámbiate de carrera", no es el tipo de respuesta que uno se piensa que le van a dar al entrar en la universidad. Y, pese a que la gran mayoría de profesores te motivan y te dicen "sigue adelante que lo consigues", acaban sobresaliendo más los comentarios negativos que los positivos.

Otro de los problemas con los que me he encontrado ha sido con los proyectos docentes. No creo que se hayan adaptado al cien por cien a lo que el Plan de Bolonia estipulaba. Hemos pasado de lo ideal de este Plan, con una metodología ideal de trabajos en la que puedo descansar cuando lo necesite y organizarme como quiera, a realizar exámenes en un tiempo determinado, prácticas obligatorias 
y trabajos grupales. Se ha evolucionado de tener mucho trabajo y poderlo organizar como nosotros quisiéramos y a poderlo hacer, más o menos, con un campo de actuación más abierto a tener más trabajo si cabe, con asistencias obligatorias que luego no se valoran y con un seguimiento más estricto.

Lo que necesito es más libertad para yo organizar mi tiempo. Y en realidad, funciona, si tú te puedes organizar a tu manera, lo acabas sacando todo adelante. El problema es ese, que hemos pasado de un modelo muy flexible que estaba en prueba a un modelo muy rígido que no te permite hacer nada. Además, en los proyectos docentes no se especifica nada sobre adaptaciones de la materia, son muy genéricos y únicamente se limitan a indicar el temario y el contenido que se va a trabajar en la asignatura.

Entiendo que el contenido ha de ser el mismo para todo el mundo, la universidad no puede hacer una selección de contenidos ni indicar las adaptaciones concretas ya que la diversidad funcional es muy heterogénea y en el plan docente no se puede contemplar todas las necesidades de todo el mundo, pero sí que se puede adaptar o, al menos, indicar en el apartado específico de adaptaciones que se realizarán las medidas y actuaciones oportunas dependiendo de las necesidades del alumno o de la diversidad funcional.

A ver, yo no puedo pedir que se quiten unas prácticas, son necesarias para mi formación, pero sí puedo pedir adaptaciones y recursos para poderlas hacer. Los proyectos docentes deben especificar y contemplar, que no lo hacen, cauces y vías de comunicación y negociación con los profesores para poder llegar a un acuerdo sobre la materia, dónde yo pueda realizar lo mismo que mis compañeros, pero adaptado a mis necesidades, si no cada dos por tres tendré la misma situación de inaccesibilidad a la información y a mi formación.

En definitiva, creo que la universidad debería ayudar al estudiante para que no se encuentre señalado por determinadas circunstancias ni tenga que pedir recursos extras. Considero que el día en el que pueda ser un alumno más, con igualdad de condiciones y con una educación de calidad y equitativa entonces, ese día, diré que la universidad, por fin, es una institución que genera mecanismos de inclusión.

\section{La Historia de Vida de Rafa}

Me llamo Rafa y tengo 21 años. Vivo con mi madre y mi hermana en Dos Hermanas. Somos una familia humilde y trabajadora, todo lo que tenemos es fruto de nuestro esfuerzo y trabajo. Me considero un chico simpático y sencillo, aunque también tímido y reservado. Sin embargo, creo que tengo que presentaros a mi colega que lleva conmigo desde los 3 años de edad, la Distrofia Muscular de Duchenne. Una enfermedad genética y degenerativa que poco a poco hace que mis músculos se vayan debilitando y desaparezcan.

Mi enfermedad ha ido evolucionando a lo largo de mi vida limitando mucho mi independencia. Actualmente he perdido toda la movilidad de mi cuerpo, aunque no por ello la sensibilidad, y dependo totalmente de mi madre para cualquier actividad cotidiana: comer, moverme, vestirme, asearme... No obstante, esto no me ha hecho tener ningún tipo de complejos, a pesar de mi diversidad funcional. Yo 
siempre me he considerado una persona completamente normal, al contrario, mi enfermedad me da más fuerza para superarme cada día y ser mejor persona.

Mi primer año de universidad fue bastante negativo, vi mucho desconocimiento hacia la diversidad funcional por parte de los compañeros y falta de empatía. Ellos ya tenían ideas preconcebidas de lo que te pasa y te ocurre, y, para ser sinceros, no tienen ni idea. Algunos de ellos no querían ponerse conmigo en los trabajos porque pensaban que yo hacía menos que ellos... Algunos se han atrevido a decir que “iqué hago yo estudiando periodismo con mi diversidad funcional!” Se ve que ni me conocían ni sabían de mis limitaciones. Soy capaz de quedarme un día entero sin dormir con tal de entregar mi parte, en eso soy muy responsable.

Sé que yo soy una persona tímida y que, aunque lo esté trabajando y mejorando, me suele costar relacionarme con otras personas, pero yo me esperaba otra cosa al entrar en la facultad, como, por ejemplo, gente más responsable. Pero lo que vi no tiene nada que ver con lo que pensaba. Esto me hizo desmotivarme y empecé a faltar a clase porque no veía ningún tipo de compañerismo. Pero bueno, un compañero me facilitó los apuntes y en verano me puse a estudiar como un loco: aprobé cuatro de cinco.

Además, unido a la falta de compañerismo me encontré con la barrera del profesorado, quien, al verme allí, no sabía ni conocía exactamente mi situación y el motivo del por qué mi madre tenía que estar allí. Hubo profesores que incluso me lo cuestionaban y no lo entendían, para ellos, debía de solicitar ayuda a la Unidad de Atención a Estudiantes con Discapacidad. Se ve que no conocían bien mi situación.

Ya conforme iban pasando los días y me iban viendo en clase, poco a poco, parece que iban comprendiendo mis necesidades, pero esto es como todo: hay profesores que te prestan toda la ayuda posible, te facilitan los recursos para poder estudiar e intentan darte unos conocimientos para que después pueda aplicarlo a mi profesión. Pero hay otros profesores que, aún pasando años y explicándoles mis necesidades, no me facilitan nada.

Por ejemplo, cuando he tenido que realizar alguna actividad en clase y la hemos tenido que entregar, ha sido mi madre la que la ha realizado y, los profesores, cuando no conocían mi situación pues me suspendían la tarea o me llamaban la atención porque las entregaba con faltas de ortografía. Pero claro, es mi madre la que lo escribe ¡demasiado hace ya! Entonces, cuando los profesores conocieron la situación o bien me adaptaban la actividad o me dejaban entregarla con las faltas, sin penalizarme por ello.

Sin embargo, también tengo que hacer mención a los profesores que me han ayudado, tanto personalmente como académicamente. En especial, a mi profesora María, con la que llevo dos cursos. Me ha adaptado todo lo que he necesitado. Si por problemas de salud he faltado, ella ha realizado conmigo tutorías y me ha solventado las dudas que pudiera tener, me ha atrasado entregas, me ha modificado fechas de exámenes, etc. Aunque en ocasiones tenga que faltar a clase, ella entiende mi situación y sabe que voy a la facultad porque quiero y es mi vocación, no estoy allí para perder el tiempo. En fin, solo puedo decir que ojalá la universidad tuviera más profesores como ella, con esa gran humanidad, empatía y concienciación. 
Por otro lado, el profesor de Producción Periodística también me ha apoyado bastante. Una de las actividades que tuvimos que realizar era un blog ${ }^{5}$ donde teníamos que colgar diez noticias. Al tener problemas para redactar y estar un poco desubicado, perdido y desmotivado, él me puso en contacto con un periodista que me ayudó en la tarea y, la verdad, es que me fue bastante bien.

En general, puedo decir que he echado en falta asesoramiento y orientación a los profesores sobre las necesidades de las personas con diversidad funcional porque entiendo que no vas a saber de todas, pero cuando la universidad conoce que hay un alumno con determinadas necesidades en un aula debería preparar y formar a los docentes.

\section{DISCUSIÓN}

Diferentes universidades, tanto nacionales como internacionales, manifiestan la importancia y necesidad de que el alumnado con diversidad funcional participe y se encuentre incluido en las instituciones de enseñanza superior en igualdad de condiciones que el resto de sus compañeros. Tal y como indica Fernández Batanero (2011), el Espacio de Educación Superior se basa en un cambio de paradigma desde donde se propone un aprendizaje basado en la excelencia y donde el profesor se convierta en una guía para el alumnado. Dentro de este contexto, los participantes de esta investigación destacaron como un hito fundamental al profesorado para garantizar prácticas educativas inclusivas. Este trabajo revela datos importantes en los que se remarca la necesidad imperante de una actitud positiva hacia la diversidad funcional por parte del profesorado, fomentando prácticas inclusivas, así como la utilización de diferentes metodologías que no sean exclusivamente las lecciones magistrales. Para ello se requiere de la realización de modificaciones curriculares, donde se empleen varios y diferentes recursos didácticos para enseñar, usando las nuevas tecnologías de forma didáctica y formándose en temas relacionados con la diversidad funcional.

Si nos centramos en los datos obtenidos en esta investigación, podemos concluir que existen más barreras que oportunidades de aprendizaje creadas por parte del profesorado. Los participantes expresaron que el apoyo recibido por parte de los docentes, en ocasiones, era debido a su buena voluntad ejerciendo un impacto positivo en su motivación personal y en su rendimiento académico. Sin embargo, estas actitudes positivas eran más difíciles de conseguir ya que dependían de la actitud y voluntad del profesorado (Tinsley \& Beale, 2010).

Por el contrario, en el entorno universitario estas actitudes no son siempre las más adecuadas, ya que los participantes del estudio comentaban cómo determinados profesores no dieron respuesta a sus problemas, no plantearon soluciones, no realizaron adaptaciones y le ofrecieron los recursos necesarios y, en algún caso en concreto, las tutorías fueron excesivamente rígidas y estandarizadas.

Los resultados obtenidos en este trabajo son coincidentes con investigaciones previas (Fuller et al., 2004; Hadjikakou \& Hartas, 2008; Konur, 2002; Nielsen, 2001; Tinklin et al., 2004). También en estos trabajos, en ocasiones, algunos profesores no se mostraban dispuestos a realizar ajustes razonables, ya que consideraban que bajaban el nivel de enseñanza o suponía un trato de favor frente a otros 
compañeros. Asimismo, también en investigaciones como las de Borland y James (1999) y Moswela y Mukhopadhyay (2011), se obtuvieron conclusiones similares a las que hemos llegado en este artículo. En estos trabajos se indicaban que las barreras actitudinales eran las que mayor impacto ejercían sobre el aprendizaje y el rendimiento académico de los estudiantes.

Resulta de especial interés que los hallazgos obtenidos en este estudio relacionados con las barreras identificadas por los estudiantes (temarios de las asignaturas excesivamente amplios; rigidez del profesorado a la hora de enseñar; relaciones no cercanas con el alumnado; falta de empatíal nada tienen que ver con la diversidad funcional, sino que pueden ser cuestiones que también afectan al resto de estudiantes. Trabajos como los de Castro de Paz et al., (2006), Fuller et al. (2004), González et al., (2006) o Jacklin, Robinson, O’Meara y Harris (2007), llegaron también a resultados similares. No obstante, no podemos olvidar que, para este colectivo, estas cuestiones son más complejas que para el resto, teniendo en ocasiones que pasar por una carrera de obstáculos (Foreman et al., 2001).

Otro de los temas que nos gustaría resaltar es el sobresfuerzo que estos estudiantes tuvieron que realizar para poder superar sus retos académicos. Para la mayoría de ellos, la universidad les aportó nuevas oportunidades en la vida (Prowse, 2009) y nuevos caminos laborales. Sin embargo, este camino, en ocasiones, no fue fácil. Para ellos, sus trayectorias educativas y vitales se encontraron marcadas por obstáculos y situaciones que debían ir solventand o y, pese a que consiguieron los objetivos establecidos, el trabajo que tuvieron que realizar para ello fue duro y largo en el tiempo. Por lo que, su proceso de resiliencia se basó en la creación de estrategias de adaptación, organización y estudio como fundamentales para adaptarse al contexto universitario, en ocasiones adverso (Ewert \& Yoshino, 2011; Hartley, 2011; Sandín et al., 2014).

Consideramos que a pesar de que los cambios y transformaciones que se tienen que realizar en las universidades son complejas y profundas, en ocasiones, son modificaciones sencillas, como facilitar el material por adelantado, adaptar las diapositivas, utilizar recursos que permitan el acceso a la información, permitir las grabaciones de audio en las clases o informar con antelación al profesorado de las necesidades de sus alumnos. Sin embargo, estas cuestiones que a simple vista parecen asequibles, en ocasiones, suponen una gran barrera para algunos estudiantes cuando el profesorado no muestra una actitud positiva ni empática por emprender estas iniciativas.

Respecto de las prácticas educativas y metodologías docentes, los participantes del estudio recomendaron que el profesorado se actualizara y utilizara metodologías diferentes a la lección magistral, a favor de metodologías más participativas y activas donde se incluyera al alumnado.

Con relación a los materiales del curso y al acceso de los mismos, los estudiantes plantearon serias barreras para acceder a los mismos, y especialmente, para el acceso a la información. Destacaron que el profesorado no tenía el material preparado ni adaptado con antelación. En este sentido, en el caso de Andrés con su diversidad visual se hizo mucho más serio y grave su problema, ya que no podía tener los apuntes o los tenía más tarde que sus compañeros. Para solventar este problema, entre las posibles soluciones que se plantearon, se señaló la aportación de la bibliografía de la asignatura por adelantado. 
Por último, nos gustaría resaltar que actualmente existen estrategias para dar respuesta a este tipo de barreras y que fomentan el aprendizaje inclusivo: trabajo por proyectos, aprendizaje cooperativo, enseñanza multinivel, entre otras. Además, trabajos como los Watchorn, Larkin, Amg y Hitch (2013) hablan sobre las experiencias de los estudiantes recomendando el uso de las prácticas diseñadas basadas en los principios del diseño universal (Dell, Dell \& Blackwell, 2015). La implementación de prácticas docentes basadas en los principios del diseño universal de aprendizaje podría suponer en un futuro la eliminación de barreras en el aprendizaje, no solo al alumnado con diversidad funcional, sino también, al resto de estudiantes. En esta línea, estamos de acuerdo con Riddell et al. (2005) o Shaw (2009) quienes indican que, si las prácticas de enseñanza mejoran para los estudiantes con diversidad funcional, revertirán positivamente tanto en la enseñanza como en el aprendizaje de todos los alumnos.

\section{REFERENCIAS}

Aguirre, R. \& Miguel, C. (2011). Estudiantes inmigrantes con diversidad funcional en la Universidad Complutense de Madrid: nuevos retos para la intervención del Trabajo Social. Revista de Trabajo y Acción Social, 50, 198-214. Recuperado de https://goo.gl/d8Ki13

Alcain, E. \& Medina, M. (2017). Hacia una educación universitaria inclusiva: realidad y retos. Revista Digital de Investigación en Docencia Universitaria, 11(1). doi: http://dx.doi.org/10.19083/ridu.11.530

Alonso, A. \& Díez, E. (2008). Universidad y discapacidad: Indicadores de buenas prácticas y estándares de actuación para programas y servicios.

Revista Española sobre Discapacidad Intelectual, 39(2), 82-98. Recuperado de https://goo.gl/NMyXZd

Bertaux, D. (2005). Los relatos de vida. Barcelona: Bellaterra.

Boletín Oficial del Estado (13 de abril de 2007). Ley Orgánica 4/2007, de 12 de abril, por la que se modifica la Ley Orgánica de 6/2001, de 21 de diciembre, de Universidades. Recuperado de https://goo.gl/glELho

Boletín Oficial del Estado (3 de diciembre de 2013). Real Decreto Legislativo 1/2013, de 29 de noviembre, por el que se aprueba el Texto Refundido de la Ley General de derechos de las personas con discapacidad y de su inclusión social. Recuperado de https://goo.gl/uMBTXk Bolívar, A., Domingo, J. \& Fernández, M. (2001). La investigación biográfico-narrativa en educación. Enfoque y metodología. Madrid, La Muralla. Borland, J. \& James, S. (1999). The Learning Experience of Students with Disabilities in Higher Education. A case study of a UK university. Disability \& Society, 14(1), 85-101. doi: http://dx.doi.org/10.1080/09687599926398

Castro de Paz, J. F., Llorca Llinares, M., Álvarez Pérez, P. \& Álvarez Durán, D. (2006). Universidad y Diversidad: Necesidades Docentes en la atención al alumnado con discapacidad. Revista Qurriculum, 19, 189-209. Recuperado de https://goo.gl/IFdS88

Cerrillo, R., Izuzquiza, D. \& Egido, I. (2013). Inclusión de jóvenes con discapacidad intelectual en la Universidad. Revista de Investigación en Educación, 11(1), 41-57. Recuperado de https://goo.gl/txYd1w

Cotán, A. (2015). Enseñanza Superior y Educación Inclusiva: múltiples miradas desde las historias de vida de estudiantes con discapacidad (Tesis doctoral) Universidad de Sevilla, Sevilla. Recuperado de https://idus.us.es/xmlui/handle/11441/32051

Dalmau, M., Llinares, M. \& Sala, I. (2013). Formación universitaria e inserción laboral. Titulados españoles con discapacidad y competencias profesionalizadoras. Revista Española de Discapacidad, 1(2), 95-118. Recuperado de https://goo.gl/pCA4WH

Debrand, C. \& Salzberg, S. (2005). A Validated Curriculum to Provide Training to Faculty Regarding Students with Disabilities in Higher Education. Journal of Postsecondary Education and Disabilities, 18(1), 49-62. Recuperado de https://goo.gl/dSCYgH

Dell, C. A., Dell, T. F. \& Blackwell, T. L. (2015). Applying universal design for learning in online courses: pedagogical and practical considerations. The Journal of Educators Online-JEO, 13(2), 166-192. Recuperado de https://goo.gl/UrZXIY

Denhart, H. (2008). Deconstructing Barriers: Perceptions of Students Labelen with Learning Disabilities in Higher Education. Journal of Learning Disabilities, 41(6), 483-497. doi: http://dx.doi.org/10.1177/0022219408321151

Denzin, N. K. \& Lincoln, Y. S. (1998). The landscape of qualitative research. New Delhi: Sage Publications. 
Doktor, J. (2010). Promoting inclusive classrooms: the mutuality of interests between profesional development school partners. SchoolUniversity Partnerships, 4(1), 7-14. Recuperado de https://goo.gl/iEgld1

Doughty, H. \& Allan, J. (2008). Social capital and the evaluation of inclusiveness in Scottish further education colleges. Journal of Further and Higher Education, 32(3), 275-284. doi: http://dx.doi.org/10.1080/03098770802220454

Escandell, M. O., Dolcet, A. M., Alonso, J., Cabello, M. D., Cabrera, M. D., Díaz, G., \& Naval, A. (2008). Cooperación institucional para la integración: actividades de sensibilización social sobre discapacidad visual en la Universidad de Las Palmas de Gran Canaria. Revista sobre ceguera y deficiencia visual, 54, 17-22. Recuperado de https://goo.gl/DGGFvU

Ewert, A. \& Yoshino, A. (2011). The influence of short-term adventure-based experiences on levels of resilience. Journal of Adventure Education and Outdoor Learning, 11(1), 35-50. doi: http://dx.doi.org/10.1080/14729679.2010.532986

Fernández Batanero, J. Ma (2011). Competencias docentes para la inclusión del alumnado universitario en el marco del Espacio Europeo de Educación Superior. Revista de Educación Inclusiva, 4(2), 135-146. Recuperado de https://goo.gl/kHvC7M

Foreman, P., Dempsey, I., Robinson, G. \& Manning, E., (2001). Characteristics, academic, and post-university outcomes of students with a disability at the University of Newcastle. Higher Education Research \& Development, 20(3), 313-325. doi: http://dx.doi.org/10.1080/07294360120108386

Fuller, M., Bradley, A. \& Healey, M. (2004). Incorporating disabled students within an inclusive higher education environment. Disability \& Society, 19(5), 455-468. doi: http://dx.doi.org/10.1080/0968759042000235307

Fuller, M., Healey, M. Bradley, A. \& Hall, T. (2005). What are disabled students' experiences of learning at university? Social Diversity and Difference. Recuperado de https://goo.gl/545w6U

Gibson, S. (2012). Narrative accounts of university education: socio-cultural perspectives of students with disabilities. Disability \& Society, 27(3), 353-369. doi: http://dx.doi.org/10.1080/09687599.2012.654987

Goetz J. P. \& LeCompte, M. D. (1988). Etnografía y diseño cualitativo en investigación cualitativa. Madrid: Morata.

González Alfonso, M. C., Guzmán Rosquete, R., Sánchez Rodríguez, J. \& González García, D. (2006). Estudiantes discapacitados en la Universidad: Percepciones sobre las respuestas a sus necesidades educativas. Revista Qurriculum, 19, 173-188. Recuperado de https:// goo.gl/pdHfeT

Guasch, D. \& Hernández, J. (2011). Observatorio Universidad y Discapacidad. Principios de igualdad de oportunidades y Accesibilidad Universal en los Planes de Estudios de los títulos de Grado de las universidades españolas. Barcelona: Observatorio Universidad y Discapacidad.

Hadjikakou, K. \& Hartas, D. (2008). Higher education provision for students with disabilities in Cyprus. Higher Education, 55(1), 103-119. doi: http://dx.doi.org/10.1007/s10734-007-9070-8

Hammersley, M. \& Atkinson, P. (1994). Etnografía. Métodos de investigación. Barcelona: Paidós.

Hartley, M. T. (2011). Examining the Relationships Between Resilience, Mental Health, and Academic Persistence in Undergraduate College Students. Journal of American College Health, 59(7), 596-604. doi: http://dx.doi.org/10.1080/07448481.2010.515632

Healey, M., Fuller, M., Bradley, A. \& Hall, T. (2006). Listening to students: the experiences of disabled students of learning at university. En M. Adams \& S. Brown (Eds.), Inclusive learning in higher education: improving provision for disabled students (pp. 32-43). London: Routledge Falmer.

Hopkins, L. (2011). The path of least resistance: a voice-relational analysis of disabled students experiences of discrimination in English universities. International Journal of Inclusive Education, 15(7), 711-727. doi: http://dx.doi.org/10.1080/13603110903317684

Jacklin, A., Robinson, C., O'Meara, L., \& Harris, A. (2007). Improving the experiences of disabled students in higher education. Recuperado de https://goo.gl/RHEjh2

Konur, O. (2002). Assessment of Disabled Students in Higher Education: Current public policy issues. Assessment \& Evaluation in Higher Education, 27(2), 131-152. doi: http://dx.doi.org/10.1080/02602930220128715

Leyser, Y. \& Greenberger, L. (2008). College students with disabilities in teacher education: faculty attitudes and practices. European Journal of Special Needs Education, 23(3), 237-251. doi: http://dx.doi.org/10.1080/08856250802130442

Moriña, A. (2017a). Inclusive education in higher education: challanges and opportunities. European Journal of Special Needs Education, 32(1), 3-17. doi: http://dx.doi.org/10.1080/08856257.2016.1254964

Moriña, A. (2017b). Investigar con Historias de Vida. Metodología biográfico-narrativa. Madrid: Narcea.

Moriña, A. (en prensa). Formación del profesorado para una educación inclusiva en la universidad. Madrid: Síntesis.

Moriña, A.; Cortés, Ma D. \& Molina, V. (2015). What if we could imagine the ideal professor? Proposals for improvement by university students with disabilities. Teaching and Teacher Education, 52, 91-98. doi: http://dx.doi.org/10.1016/j.tate.2015.09.008

Moswela, E. \& Mukhopadhyay, S. (2011). Asking for too much? The voices of students with disabilities in Botswana. Disability \& Society, 26(3), 307-319. doi: http://dx.doi.org/10.1080/09687599.2011.560414 
Mullins, L. \& Preyde, M. (2013). The lived experience of students with an invisible disability at a Canadian university. Disability \& Society, 28(2), 147-160. doi: http://dx.doi.org/10.1080/09687599.2012.752127

Murray, C., Lombardi, A. \& Wren, C. T. (2011). The Effects of Disability-Focused Training on the Attitudes and Perceptions of University Staff. Remedial and Special Education, 32(4), 290-300. doi: http://dx.doi.org/10.1177/0741932510362188

Nielsen, J. A. (2001). Successful University Students with Learning Disabilities, Journal of College Student Psychotherapy, 15(4), 37-48. doi: http://dx.doi.org/10.1300/J035v15n04_05

Novo, I., Muñoz, J. M. \& Calvo, C. (2011). Análisis de las actitudes de los jóvenes universitarios hacia la discapacidad: Un enfoque desde la teoría de la acción razonada. Relieve, 17(2), 5. Recuperado de https://goo.gl/KSZnRd

Opazo, H. (2011). Ética en investigación: desde los códigos de conducta hacia la formación den sentido ético. Revista Iberoamericana sobre Calidad, Eficacia y Cambio en Educación, 9(2), 62-78. Recuperado de https://goo.gl/1EtH7E

Pacheco, D. (2012). Nuevos alcances de la ética en investigación a partir de la ética de la liberación. Revista Reflexiones, 91(1), 117-125. Recuperado de https://goo.gl/yLMxiG

Patton, M. Q. (1987). How to use qualitative methods in evaluation. London: Sage Publications.

Prowse, S. (2009). Institutional construction of disabled students. Journal of Higher Education Policy and Management, 31(1), 89-96. doi: http://dx.doi.org/10.1080/13600800802559302

Pujadas, J. J. (2002). El método biográfico: El uso de las Historias de Vida en Ciencias Sociales. Madrid: Centro de Investigaciones Sociológicas. Richaud, MaC. (2007). La ética en la investigación psicológica. Enfoques 19(1-2), 5-18. Recuperado de https://goo.gl/LKo7IC

Riddell, S., Tinklin, T. \& Wilson, A. (2005). Disabled students in Higher Education. London: Routledge.

Rodríguez, A. \& Álvarez, E., (2014). Estudiantes con discapacidad en la Universidad. Un estudio sobre su inclusión. Revista Complutense de Educación, 25(2), 457-479. doi: http://dx.doi.org/10.5209/rev_RCED.2014.v25.n2.41683

Ruiz Hidalgo, C. (2015). Tutorización de un alumno con síndrome de asperger: coordinación y evaluación de los estudios de Grado en Derecho. Revista de Educación y Derecho, 11, 1-17. Recuperado de https://goo.gl/ayzaNA

Salvador, F. (2003). Actitudes sociales ante la discapacidad. En R. Casado \& A. Cifuentes (Eds.). El acceso al empleo y a la universidad de las personas con discapacidad. Barreras y alternativas (pp.19-34). Burgos: Servicio de Publicaciones de la Universidad de Burgos.

Sandín, Ma P., Bazoco, J., Del Campo, J., Forés, A., Massot, I., Sacramento, R., \& Sánchez, A. (2014). Resiliència, immigraciò i èxit escolar. Temps d'Educació, 46, 11-31. Recuperado de https://goo.gl//7MINM

Shaw, J. A. (2009). Chance happenings in life and psychotherapy. Psychiatry, 72(1), 1-12. doi: http://dx.doi.org/10.1521/psyc.2009.72.1.1

Sikes. P. (2006). On dodgy ground? Problematics and ethics in educational research. International Journal of Research and Method in Education, 29(1), 105-117. doi: http://dx.doi.org/10.1080/01406720500537502

Strauss, A.L. \& Sales, A. (2010). Practice Brief. Bridging the gap betweem Disability studies and Disability services in higuer Education: A model center on Disability. Journal of Postsecundary Education and Disability, 23(1), 79-84. Recuperado de https://goo.gl/wT0atr

Stutchbury, K. \& Fox, A. (2009). Ethics in educational research: introducing a methodological tool for effective ethical analysis. Cambridge Journal of Education, 39(4), 489-504. doi: http://dx.doi.org/10.1080/03057640903354396

Teachability (2002). A curriculum for disabled pupils. Glasgow: University of Strathclyde.

Thomas, L. (2016). Developing inclusive learning to improve the engagement, belonging, retention, and success of students from diverse groups. En M. Shah, A. Bennett \& E. Southgate (Eds.), Widening higher education participation. A global perspective (pp. 135-159). 0xford: Elsevier.

Tinklin, T., Riddel, S. \& Wilson, A. (2004). Policy and provision for disabled students in higher education in Scotland and England: the current state of play. Studies in Higher Education, 29(5), 637-657. doi: http://dx.doi.org/10.1080/0307507042000261599

Tinsley, B. \& Beale, M. (2010). High hope and low regard: The resilience of adolescents' Educational Expectations while developing in challenging political contexts. Research in Human Development, 7(3), 183-201. doi: http://dx.doi.org/10.1080/15427609.2010.505780

Vain, P.D. (2011). La , ICE, Revista Electia como naturalizacicia y Cambio en Educacios que se puedan obtener y que puedan dañar a los dempectos ciética en la investigación educativa y el riesgo del uso de la ciencia como naturalización de lo social. REICE, Revista Electrónica Iberoamericana sobre Calidad, Eficacia y Cambio en Educación, 9(2), 15-23. Recuperado de https://goo.gl/jRnntb

Vander Kloet, M. (2015). Accessibility in Teaching Assistant Training: A Critical Review of Programming from Ontario's Teaching and Learning Centres. The Canadian Journal for the Scholarship of Teaching and Learning, 6(2), 9. doi: http://dx.doi.org/10.5206/cjsotl-rcacea.2015.2.9

Vickerman, P. \& Blundell, M. (2010). Hearing the voices of disabled students in higher education. Disability \& Society, 25(1), 21-32. doi: http:// dx.doi.org/10.1080/09687590903363290

Watchorn, V., Larkin, H., Ang, S. \& Hitch, D. (2013). Strategies and effectiveness of teaching universal design in a cross-faculty setting. 
Teaching in Higher Education, 18(5), 477-490. doi: http://dx.doi.org/10.1080/13562517.2012.752730

Wessel, R. D., Jones, D., Blancj, C. L. \& Markle, L. (2015). Pre-enrollment considerations of undergraduate wheelchair users and their postenrollment transitions. Journal of Postsecondary Education and Disability, 28(1), 57-71. Recuperado de https://goo.gl/Q3nHUf

Yssel, N., Pak, N. \& Beilke, J. (2016). A Door Must Be Opened: Perceptions of Students with Disabilities in Higher Education. International Journal of Disability, Development and Education, 63(3), 384-394. doi: http://dx.doi.org/10.1080/1034912X.2015.1123232

(c) Los autores. Este artículo es publicado por la Revista Digital de Investigación en Docencia Universitaria del Área de Institutional Research and Effectiveness de la Dirección de Aseguramiento de la Calidad, Universidad Peruana de Ciencias Aplicadas. Este es un artículo de acceso abierto, distribuido bajo los términos de la LicenciaCreativeCommons Atribución-Compartirlgual 4.0 Internacional.l http://creativecommons.org/licenses/bysa/4.0/I, que permite el uso no comercial, distribución y reproducción en cualquier medio, siempre que la obra original sea debidamente citada. 